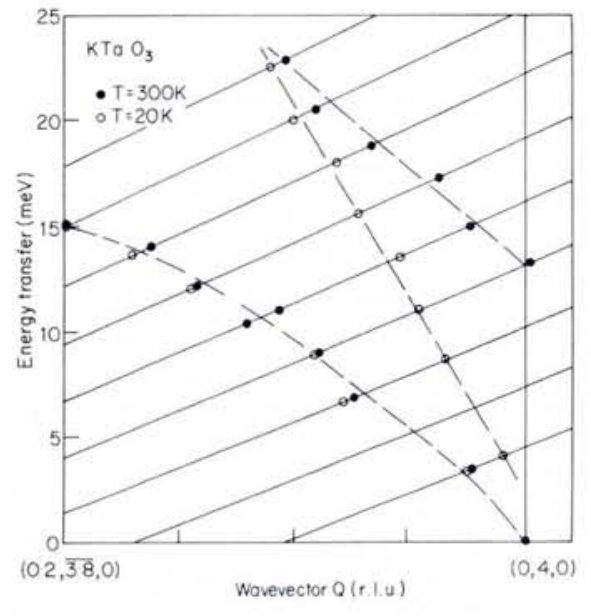

Fig. 7 - The dispersion relation for $\mathrm{KTaO}_{3}$ measured along the [110] direction about the (040) reciprocal lattice point. The full circles correspond to $300 \mathrm{~K}$ and the open circles to $20 \mathrm{~K}$. The observed shift of the dispersion curve of the zone centre TO, mode phonons indicates an increase in the amplitude of the corresponding atomic motions as a phase transition is approached on reducing the temperature.

\section{Edgar Lüscher}

Professor Edgar Lüscher, Chairman of the Metals Section of the EPS Condensed Matter Division, died after a long cruel disease on 16 January 1990.

He was born in Switzerland in 1925 and studied mathematics and physics at the Swiss Federal Institute of Technology in Zürich, and at the University of Lausanne. He received his diploma in 1949, a Ph.D. in physics in 1955 and obtained his habilitation at the University of Lausanne a year later. After teaching and then working for several years in industry, he became a professor at the University of Illinois, Urbana. Since 1964 until his tragic death he was Full Professor of Experimental Physics at the Technical University, München. He was elected in 1965 as member of the Max Planck Society.

His main research fields spanned atomic and condensed matter physics, particularly liquid and amorphous metals. His more recent interests were primarily oriented towards biophysics and chaotic phenomena in general.

We deeply mourn his death, both as a scientist and as a great humanist.

\section{F. Cyrot-Lackmann,}

Grenoble

\section{CORRECTION}

In last month's Directory issue we inadvertently designated E. Jakeman instead of $\mathrm{H}$. Ryde as the Vice-Secretary of the Executive Committee. towards the phase transition, the lower phonon energy means that the amplitude of the corresponding atomic motions increases. As the phonon energy reaches zero at a particular wave vector, the amplitudes of the atomic motions tend to diverge and the phase transition takes place.

\section{Conclusion}

The PRISMA spectrometer has already given encouraging evidence of its potential to measure coherent excitations and the onset of phase transitions at low temperatures. Time will tell if it is eventually able to equal the scientific success of the triple axis spectrometer.

\section{Acknowledgement}

Some of this work formed parts of the scientific programmes of the United Kingdom Atomic Energy Authority, the SERC, and the Italian Consiglio Nazionale delle Richerche.

\section{REFERENCES}

(1) Windsor C.G., Proposal for a High Symmetry Spectrometer, paper submitted to the Spallation Neutron Source Science Planning Group, October 1977.

(2) Andreani C., Carlile C.J., Cilloco F., Petrillo C., Sacchetti F., Stirling G.C. and Windsor C.G., Nucl. Inst. Meth. A254 (1987) 333.

(3) Steigenburger U. et al., Nucl. Inst. Meth. (submitted for publication).

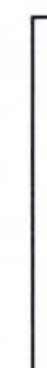

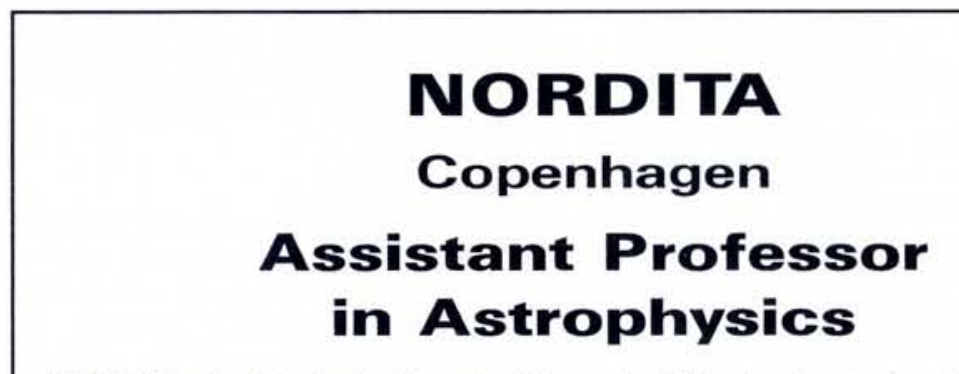

NORDITA, the Nordic Institute for Theoretical Physics, located at the Niels Bohr Institute of Copenhagen University, has an opening for an Assistant Professor in theoretical astrophysics starting in September 1991, or some other date to be agreed upon.

NORDITA is supported by the five Nordic countries, Denmark, Finland, Iceland, Norway and Sweden. Research at the Institute is at present carried out mainly in astrophysics and cosmology, complex systems (including neural nets), condensed matter physics, high energy physics and nuclear physics. There are thus good opportunities to carry out cross-disciplinary studies. Staff members working in astrophysics are K. Enqvist, B. Pagel and C.J. Pethick, whose main interests are in cosmology, the chemical evolution and dynamics of galaxies, high energy astrophysics, and neutron stars. Astrophysicists at NORDITA have close contacts with those at the Niels Bohr Institute, the Danish Space Research Institute, the Copenhagen University Observatory and other institutes in the Nordic area. There are also opportunities for observing at the European Southern Observatory and the Nordic Optical Telescope, among other facilities.

The scientific staff includes six positions as permanent professors, four positions as assistant professors, and Nordic assistant professors. In addition there is a fellowship programme for Nordic graduate students and postdocs. The Institute's activities include an extensive visitor programme for scientists from all over the world, and symposia and summer schools arranged either by NORDITA itself or in cooperation with other Nordic institutes.

The successful applicant is expected to guide fellows at roughly the postdoctoral level, to interact with colleagues at NORDITA and elsewhere in the Nordic countries and to take an active part in the organization of meeting and courses. The position provides excellent opportunities to pursue original research and to have contact with a wide range of developments in theoretical physics. There are good facilities for travelling to other institutes and to meetings, and the Assistant Professor will be encouraged to invite guest scientists to visit NORDITA.

The initial appointment will be for three years, with the possibility of renewal up to a total of six years. The annual salary will be in the range of $240000-310000$ Danish kroner depending on experience.

Those interested in the appointment should send a curriculum vitae, a list of publications and the names of three referees before 1 November 1990 to:

Prof. C.J. Pethick, Director, NORDITA, Blegdamsvej 17, DK-2100 Copenhagen 0. Tel.: ++45-31 4216 16, Fax: ++45-31 3891 57, Email: nordita@nbivax.nbi.dk.

There is no restriction on the nationality of the applicant. Those wishing to recommend suitable candidates are urged to contact the Director. 